Vojislav B. Krstić

Božidar V. Krstić ${ }^{1}$

\section{EFFECTIVENESS OF PREVENTIVE MAINTENANCE OF MOTOR VEHICLES}

Abstract: There is given methodology of determination the periodicality conveymg processes of preventive maintenance vehicle, in limit the strategy its preventive maintenance. This methodology is possibly apply when there can convey the revision which doesn't change intensity falilure of analysed part after every falilure.

Key words: vehicle, preventive meintenance

\section{Introduction}

The effectiveness of preventive maintenance of motor vehicles represents the timely identification of preventive operations (works) and the quality of their implementation. The time of preventive operations determines the speed of the occurrence of a malfunction on the vehicle. The quality of the detection of malfunction depends on the convenience of the applied forecasting methods, the way of finding faulty elements and the time predicted for preventive surgery. It is indisputable that in one case and in the other case the effectiveness of preventive maintenance is essentially influenced by the skill of using a motor vehicle. Here are some of the basic methods for preventing failure with their characteristics.

Controlling the change in the physicochemical structure of the elements of the device, which is applied in case the elements of the structure change are determined for the element with time (when the forecasting parameter is known) and if there are forecasting devices. - Control the quality of the device performance based on the output parameter. This method is based on the fact that the change in the structure of the input elements leads to a disturbance of functionality, i.e. conditional changes to the output parameter. Unlike the first method, in this case, the element that led to the malfunction can not be immediately detected. In order to find fault, it is necessary to find a defective part and repair it.

Use statistical distribution of the probability of correct work of parts until the first failure, obtained on the basis of long-term use of the device. It is possible, with a certain probability, to predict the moment of failure of the element and take measures for its prevention. This method is usually applied when there is no possibility to control the change of the structure of the elements or there is no dependence between the change in the structure and the output parameter of the device. The mentioned methods are most often used to prevent the failure of electromechanical and mechanical circuits, and the elements of motor vehicles for which the statistical legality of the failure occurred. Usually, an

\footnotetext{
${ }^{1}$ Corresponding author: Vojislav B. Krstić

Email: bkrstic@kg.ac.rs
} 
incomplete (shortened) normal law is applied to distributing the reliability time of these elements.

In the process of using modern motor vehicles of the system (with a lot of embedded electronic systems), there are irregularities that can not be detected by any of the above methods. These malfunctions lead to inevitable failures. It is a natural assumption that preventive works in one way or another are based on the methods of detecting and remedying the aforementioned.

\section{Types of preventive works}

As a rule, for a number of technical systems, which means for motor vehicles, the following typical preventive maintenance phases can be distinguished, and each of them contains certain types of operations. The external inspection of the motor vehicle contains: disassembly, inspection of joints and connections; cleaning of elements and assemblies of the device; replacement of lubricants, checking the quality of assembly, cables and possible leaks; checking the accuracy of the operation of individual vehicle assemblies using control-measuring accessories.

Preliminary tests of the motor vehicle circuit in the operating mode include: establishing a working regime; adjustment and finishing of individual parts and assemblies; check the functioning of parts and assemblies in normal and special operating modes. Implementation of preventive control of operation of parts and assemblies of vehicles includes: assembly and completion of vehicle assemblies; checking the functionality of vehicle assemblies in normal and special regimes; complete installation and check of basic parameters of the vehicle as a whole. During preventive operations, in each maintenance phase, defective parts are detected, replaced or repaired. In the first stage of prevention - an external examination of the vehicle - it is the detection and replacement of defective parts.

In the second phase, preventive tests of vehicles in the working regime are carried out. In doing so, the detection and repair of faulty parts is carried out only in case of inability to establish the initial operating modes or the limit of the inclusion of individual parts and assemblies using the adjustment elements. When checking the functioning of the vehicle as a whole (the third stage of prevention), which includes both finishing and adjustment according to the given technical conditions, it is also possible to seek and correct malfunctions. Then, some operations, already performed earlier, are repeated, for example: adjusting individual assemblies, checking assembly, adjusting connectors, and so on.

In each stage of vehicle maintenance, preventive operations related to finding faults are carried out, and their execution time is accidental. Analyzing the content of the mentioned preventive works we can conclude that at the basis of their performance there is one of the methods for preventing failure. For a quantitative assessment of the effectiveness of these methods, a mathematical description (display) of the process of increasing and eliminating malfunction is required.

\section{Process control of the insurance of vehicles with time}

The effectiveness of preventive maintenance depends to a large extent on the timely planning of preventive works. Knowing the legality of the inadequacy on the vehicle allows for the prompt determination of the deadlines for the implementation of preventive operations. On the one hand, their premature realization is detrimental, causing unnecessary and irrational interruptions to the operation of the vehicle. On the other hand, too long the time between preventive measures is conditioned by an increase in the number of failures due to timely improper malfunctions. It means that there is an 


\section{Interinational Qualitity Conference}

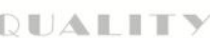
DIISSIFAIDCI

optimal periodicity of conducting preventive operations, in which the most contradictory solution is solved.

Depending on the method of preventing failure, the character of the process of accumulating defects over time can be described as follows:

1. If a forecasting parameter is known for some element, then the probability of its non-fatal operation over time is evaluated according to the following expression:

$$
P(t)=\int_{t}^{\infty} \Psi_{p 1}(t) d t
$$

where he is $\Psi_{p 1}(t)-$ function obtained by budget in the axis of time.

2. If for parts of the same type there are failures that can be prevented, and inevitable failures and are known for their statistical distribution laws over time, then the probability of failure-free operation is determined by the expression:

$$
P(t)=\int_{t}^{\infty} f(t) d t
$$

wherein

$$
f(t)=C_{1} f_{p}(t)+C_{2} f_{n}(t)
$$

$f_{p}(t), \quad f_{n}(t)-$ the density of the distribution of the probability of the occurrence of inevitable failures that can be prevented;

$C_{1}, C_{2}-$ are the coefficients that determine the percentage of each law

$f_{p}(t)$ i $f_{n}(t)$, which make the superposition $f(t)$.

Sizes $C_{1}$ and $C_{2}$ satisfy the condition:

$$
C_{1}+C_{2}=1
$$

In the first case, when a prediction parameter is known, it is usually determined certain limits of the quality of the work, which can be controlled in the process of operation. The gradual quality of work decreases as the boundary after which the dismissal falls. In this case, a preliminary degree of a controlled parameter can be determined, in which the defective element is detected and replaced. This degree is called the degree of prognosis.

In analyzing the change of the forecasting parameter over time, the following sizes are used: $\bar{\alpha}(t)-$ mathematical expectation of functions, which characterize the change with the time of the basic controlled parameter; $\bar{\alpha}_{0}-$ mathematical expectation of scattering of initial values of parameters; $\bar{\alpha}_{k r}-$ critical level of working ability (functioning) - mathematical expectation of the limit values of parameters in which a failure occurs; degree of prognosis (preventive control); $T_{p r}-$ prognosis period $\bar{\alpha}_{p r}-$ time between two preventive controls; $\quad \Delta \bar{\alpha}_{k r}, \Delta \bar{\alpha}_{p r}-\quad$ preventive controls; total and preventive parts reliability reserves, which can be challenged in the form of:

$$
\begin{gathered}
\Delta \bar{\alpha}_{k r}=\bar{\alpha}_{0}-\bar{\alpha}_{k r} \\
\Delta \bar{\alpha}_{p r}=\bar{\alpha}_{p r}-\bar{\alpha}_{k r}
\end{gathered}
$$

Knowing the statistical legality of changing a controlled parameter over time allows it to be based on the results of its measurement at the moment $\bar{t}_{k r}=\bar{t}_{p r}+T_{p r}$ is a prerequisite for preventing vehicle failure. The forecasting period depends on the rate of change of parameters with time. From this aspect, each forecasting parameter $\alpha(t)$ can be estimated by the coefficient of its change in time: 


$$
K_{\alpha}=\frac{\bar{\alpha}_{0}-\bar{\alpha}(t)}{t}
$$

which characterizes the process of multiplying defects over time.

In the second case, when the prediction parameter is unknown, the basic characteristic of the multiplication process of defects with the flow of time is the statistical distribution of the correct time to the first failure. Based on the experience in using the vehicle, it is known that the distribution of the time of the occurrence of inevitable failures, with acceptable accuracy, is approximated by the exponential by law (Babuška R, 2001):

$$
f_{N}(t)=\lambda \exp (-\lambda t)
$$

where the intensity of inevitable failures.

For disabling failures it is possible to assume the distribution described by the abridged (incomplete) normal law (Babuška R, 2001). In this case, the superposition of the above laws, according to expression (3), taking into account equations (6) and (2), will have the following form:

$$
f(t)=C_{1} \frac{c}{\sigma \sqrt{2 \pi}} \exp \left[-\frac{\left(t-T_{s r}\right)^{2}}{2 \sigma^{2}}\right]+C_{2} \lambda \exp (-\lambda t)
$$

In terms of (3) and (7), the coefficient, which determines the number of cancellations that can be prevented, represents the coefficient of the failure character. Since it is:, it turns out that:

$$
C_{2}=1-A\left(T_{e}\right)
$$

It can be shown that by replacing (7) in (2) taking into account (8), formula (2) is transformed as follows:

$$
P(t)=A\left(T_{e}\right) \frac{\Phi\left(\frac{t-T_{s r}}{\sigma}\right)}{\Phi\left(\frac{T_{s r}}{\sigma}\right)}+\left[1-A\left(T_{e}\right)\right] e^{-\lambda t}
$$

where are they: $\Phi\left(\frac{t-T_{s r}}{\sigma}\right), \Phi\left(\frac{T_{s r}}{\sigma}\right)-$ tabular functions of integral probability (Krstić B, 1999).

The expression (9) is characterized by statistical distribution during the multiplication process without the existence of a predictive parameter. If, in formula (7), the coefficients and expressions over the coefficient of the character of the failure are given a formula for determining the frequency of the phenomenon of malfunction, in the case of superposition of the exponential and the reduced normal law:

$$
\lambda_{c}(t)=\frac{A\left(T_{e}\right) \frac{c}{\sigma \sqrt{2 \pi}} \exp \left[-\frac{\left(t-T_{s s}\right)^{2}}{2 \sigma^{2}}\right]+\left[1-A\left(T_{e}\right)\right] \lambda \exp (-\lambda t)}{A\left(T_{e}\right) \frac{c}{\sigma \sqrt{2 \pi}} \int_{0}^{t} \exp \left[-\frac{\left(t-T_{s s}\right)^{2}}{2 \sigma^{2}}\right] d t+\left[1-A\left(T_{e}\right)\right] \exp (-\lambda t)} .
$$

Knowing the legality of the process of reporting defects with time (the rate of change of the forecasting parameter in the first case and the statistical distribution of the probability of operation without failure in the second case) is a prerequisite for the timely undertaking of preventive works.

\section{Discovering inquiry process}

The effectiveness of preventive work on the vehicle depends not only on the timely determination but also on the quality of their implementation, which again depends on the quality of the detection of malfunction during the implementation of preventive measures, i.e. from discovering and remedying defects. This quality depends on the expertise of the maintenance personnel, 
the quality of the equipment and the time spent. In practice, the task of determining the time of implementation of preventive operations is imposed when the level of professionalism of the staff and certain forecasting apparatus is known.

The time required for the preventive maintenance of the vehicle (and any technical system) consists of the time necessary for detection and elimination of malfunction, as well as the time required for the auxiliary works (elaboration, preparation of tools and equipment for forecasting, assembly, etc.). The time required to detect defective parts depends on the nature of the works. These works are associated with time-related processes for detecting defective parts and have a random character. The timing of their execution can be determined by the collection:

$$
T_{p c}=\sum_{i=1}^{d} t_{p i}
$$

where the timing of the implementation of the random volume of works of this type, and the number of works.

The deadlines for completing these works can not be determined in advance. In this case, the time of finding the defective element, as a rule, is much longer than the time needed to repair the defect, which means that:

Other preventive works - replacement of defective elements, disassembly, inspection, cleaning, lubrication, etc., have a determined character. The timing of their performance can be determined as follows:

$$
T_{p y}=\sum_{j=1}^{r} t_{p j}
$$

where is the number of types of works. The completion of these works can be foreseen in advance. Prevention, which includes works of random and specific character, as well as every work process, can be determined based on the frequency and duration of the work. Since the efficiency of each working process is determined based on the number of products in the unit of time and time spent for their production, so the effectiveness of the prevention can be assessed on the basis of the number of identified and remedied defects. In the realization of preventive works between the number of detected defects, the number of checked elements during the prevention and the volume of operations performed (in the general case), linear dependence can not be determined due to the random character of the detection process.

The productivity of preventive maintenance means the number of checked elements in the unit of time.

Under the intensity of the detection of defective parts, the number of discovered defective elements is understood, i.e. the foreclosures in the unit of time according to their number at the time of the time. We determine the dependence between probability and frequency of detection of defective parts and the duration of preventive work. In case of faulty detection, the frequency of detection, in analogy with the intensity of the cancellation, can be determined by the ratio of the number of detected defective parts in the unit of time, according to the number of those that have not been detected. Starting from this attitude, it turns out that: 


$$
v(t)=\frac{d n_{p v}}{\left[n_{p}\left(T_{p r}\right)-n_{p v}(t)\right] d t},
$$

wherein the number of prevented malfunctions, accumulated until the start of preventive operations; $n_{p}\left(T_{p r}\right)-n_{p v}(t)-$ is the number of faults undetected until the moment. If the numerator and the denominator of the previous formula are separated by and perform several simple transformations, it will be obtained that:

$$
v(t)=\frac{P_{p v}^{\prime}}{1-P_{p v}}
$$

After integrating (14) and transformation it turns out that:

$$
P_{p v}=1-\exp \left[-\int_{0}^{T_{p}} v(t) d t\right] .
$$

In this way, in preventive maintenance, the random process of detecting defective elements can be approximated by the acquired dependence (15). This formula corresponds to many practical cases. In an individual case, when a condition can be considered $v(t)=$ const. Equation (15) determines the exponential law of distributing the probability of detecting defective elements. This does not give a complete physical image but essentially simplifies the mathematical process. In the case where the detection of malfunction is a process that is not proper (defects are not required), the probability of failure prevention, in general, depends on the accuracy of the forecast and the time spent on that process. It can be assumed that the probability of overcoming the defective element due to the insufficient accuracy of the measuring devices and the faults of the maintenance personnel, and the probability of passing the defective element due to lack of time, $Q(L)$ independent. In this case, the probability of detection of malfunction can be expressed as an equation:

$P_{p v}=[1-\delta][1-Q(L)]$,

where are they:

$Q(L)=\left\{\begin{array}{l}1-L T_{p}, \text { pri } 0 \leq T_{p} \leq T_{p_{0}} \\ 0, \text { priT }_{p}>T_{p_{0}} .\end{array}\right.$

$T_{p_{0}}-$ the time is needed for the prevention;

$L=\frac{1}{T_{p_{0}}}-L=\frac{1}{T_{p_{0}}}-$ time standard for preventive surgery; $\delta$ - relative error in detecting malfunction.

By computing the above symbols, the formula (16) will get the following form:

$$
P_{p v}=(1-\delta) L T_{p} .
$$

Therefore, the knowledge of the legality of the process of detecting defects, dependencies (15) and (17) is an assumption for determining the timing of the transmission of preventive work.

\section{Conclusion}

The effectiveness of preventive maintenance represents the timely identification of preventive operations (works) and the quality of their implementation. The time of 


\section{Interinational Cuality Conference 1 .}

QUA IITY

preventive operations determines the speed of the occurrence of a malfunction on the vehicle. The quality of the detection of malfunction depends on the convenience of the applied forecasting methods, the way of finding faulty elements and the time predicted for preventive surgery. It is indisputable that, in either case, the effectiveness of preventive maintenance is essentially influenced by the skill of using maintenance equipment. In the analysis of preventive measures, issues related to the quantitative assessment and the comparison of different preventive maintenance methods are often posed. The mathematical models shown in the paper provide a quantitative assessment of the impact of preventive measures on the reliability of motor vehicles. When organizing vehicle maintenance, it is necessary not only to estimate profit in reliability, but also to determine the costs for which it is achieved.

Acknowledgment: Research presented in this paper was supported by Ministry of Science and Technological Development of Republic of Serbia, Grant TR 35041.

\section{References:}

Babuška R.(2001): Fuzzy and neural control, Delft University of Technology, Delft, the Netherlands

British Standard, BS5760, Part 2 (1981) Guide to the assessment of reliability, Reliability of systems, equipments and components, BSI, London

Krstić B.(1999): Analysis of the vital parts of the engine vehicles on the base of the data from exploitation, VII International scientific conference, Simulation Research in Automotive Engineering, Lublin, Polska, 1999.

Krstić B.(2003): Determination of the optimal strategy for preventive maintenance the motor engine using policriterion optimization, International Journal for Vehicle Mechanics, Engines and Transportation Systems, Mobility Vehicles Mechanics, Volume 29, Number 4

Krstić B.(2004): Analysis of the reliability of the motor vehicle brakes bands and determination the optimal periodicity for their preventive maintenance, International Tribology Conference CONAT 2004, Brasov,

\section{Vojislav B. Krstić}

Faculty of transport and traffic engineering,

University of Belgrade, Beograd,

Serbia

\author{
Božidar V. Krstić \\ Faculty of Engineering \\ University of \\ Kragujevac,Kragujevac, \\ Kragujevac, \\ Serbia \\ bkrstic@kg.ac.rs
}




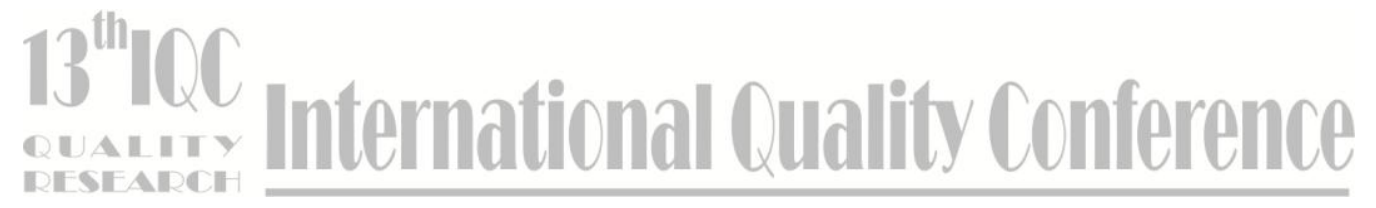

